\title{
MATRIX RATIONAL INTERPOLATION WITH POLES AS INTERPOLATION POINTS
}

\author{
M. VAN BAREL \\ Department of Computing Science, K.U.Leuven, \\ Celestijnenlaan 200A, B-s001 Heverlee, Belgium \\ B. BECKERMANN \\ Institut für Angewandte Mathematik, Universität Hannover, \\ Welfengarten 1, D-s0167 Hannover, Germany \\ A. BULTHEEL \\ Department of Computing Science, K.U.Leuven, \\ Celestijnenlaan 200A, B-9001 Heverlee, Belgium \\ and \\ G. LABAHN \\ Department of Computing Science, University of Waterloo, \\ Waterloo, Ontario, Canada
}

\begin{abstract}
In this paper, we show the equivalence between matrix rational interpolation problems with poles as interpolation points and no-pole problems. This equivalence provides an effective method for computing matrix rational interpolants having poles as interpolation points. However, this equivalence is only valid in those cases where enough pole information is known. It is an open problem on how one can transform the pole problem to a no-pole problem in other cases.
\end{abstract}

Key words: Matrix rational interpolation, pole information

\section{Introduction}

In this paper, we consider a matrix rational interpolation problem with data given at one or more interpolation points. We also allow these interpolation points to be poles of our matrix rational interpolant. In the case of the scalar pole-problem (also allowing confluent interpolation points and $\infty$ as an interpolation point), Gutknecht [5] gives an algorithm computing the solution as a continued fraction along a diagonal or a staircase in the Newton-Padé table. Additional information about scalar rational interpolants (for example concerning their existence and uniqueness) can be found in Gutknecht [6] and Stahl [8]. In [13], Van Barel and Bultheel generalize their algorithm of [9] also allowing confluent interpolation points, poles and $\infty$ as an interpolation point.

The algorithm of [13] is based on the fact that all solutions of an equivalent linearized interpolation problem can be written in terms of some basis vectors of an $\mathbf{F}[z]$-submodule of the module of all polynomial vectors $\mathbf{F}^{n}[z]$. To add a new interpolation condition, we only have to update this set of basis vectors. This can be done in a fast and reliable way leading to $O\left(k^{2}\right)$ FLOPS for $k$ interpolation conditions. For the pole-free problem, this was generalized to the vector case $[1,4$, 
$10,11]$ and also to the matrix case $[2,3,12]$. Once we have the basis vectors, we can parametrize all solutions of the original rational interpolation problem having minimal complexity [12]. In this paper, we show how to transform the pole-problem to a pole-free problem. The latter can be solved using one of the methods referred to before.

In the next section, we define the rational interpolation problem that we want to solve. This section also discusses the $\vec{s}$-McMillan degree, used as a measure of the complexity of a rational interpolant. Sections 3 and 4 introduces the concept of $(\alpha, \sigma)$-characteristics of a formal Laurent series around a point $\alpha$, and shows how they can be computed. In Section 5 , we show that our original rational interpolation problem with pole information is equivalent to another rational interpolation problem without poles as interpolation points. However, as we show in Section 6, the equivalence of the pole and the pole-free problem is only true when enough interpolation data are known at each pole. It remains an open problem how to solve the rational interpolation problem in an efficient way when this condition is not met.

\section{Rational interpolation with poles}

For the rational interpolation problem considered here, we have interpolation information at each point (possibly a pole) $\alpha \in I$, with $I$ a non-empty, finite subset of $\mathbb{F}$, F a field. We will use the following notations: $\mathbb{F}[z]$ the set of polynomials, $\mathbb{F}[[z]]_{\alpha}$ the set of formal power series around $\alpha \in \mathbf{F}, \mathbf{F}(z)$ the field of rational functions and $\mathbb{F}((z))_{\alpha}$ the field of formal Laurent series

$$
\mathbb{F}((z))_{\alpha}=\left\{\frac{1}{(z-\alpha)^{k}} \cdot f: f \in \mathbb{F}[[z]]_{\alpha}, k \in \mathbf{Z} \text { (the set of integer numbers) }\right\} .
$$

We also have the corresponding matrix versions, for example $\mathbf{F}^{p \times q}((z))_{\alpha}$ where $p, q$ are positive integers. Note that $\mathbb{F}[z] \subset \mathbb{F}(z) \subset \mathbb{F}((z))_{\alpha}$.

In the sequel, the relations $\leq, \geq$, min between integer vectors have to be understood componentwise. The vector $\vec{e}:=(1,1, \ldots, 1,1)$ and $e_{j}$ is the $j$-th column of the identity matrix.

Definition 1 The $\alpha$-order, $\alpha \in \mathbb{F}$, of $\mathbf{T} \in \mathbb{F}^{p \times q}((z))_{\alpha}$ is defined by

$$
\operatorname{ord}_{\alpha} \mathbf{T}:=\sup \left\{\sigma \in \mathbf{Z}: \mathbf{T}=(z-\alpha)^{\sigma} \cdot \mathbf{R} \text { with } \mathbf{R} \in \mathbb{F}^{p \times q}[[z]]_{\alpha}\right\},
$$

i.e., $\mathbf{R}$ is a formal matrix power series around $\alpha$. Note that $\operatorname{ord}_{\alpha} \mathbf{0}=+\infty$. For $\mathbf{T} \in \mathbb{F}^{p \times q}((z))_{\alpha}$ with rows $\mathrm{T}_{1}, \ldots, \mathbf{T}_{p} \in \mathbb{F}^{1 \times q}((z))_{\alpha}$ the $\alpha$-row order $\operatorname{rord}_{\alpha} \mathbf{T}$ is a vector with components ord $\mathrm{T}_{i}$. Similarly we define the $\alpha$-column order cord ${ }_{\alpha} \mathrm{T}$ with respect to the columns of $\mathbf{T}$.

For a vector $\vec{\sigma}=\left(\sigma_{1}, \ldots, \sigma_{m}\right)$ with components from $\mathbf{Z} \cup\{+\infty\}$ we denote $(z-\alpha)^{\vec{\sigma}}:=\operatorname{diag}\left((z-\alpha)^{\sigma_{1}}, \ldots,(z-\alpha)^{\sigma_{m}}\right)$, where $(z-\alpha)^{+\infty}:=0$. In this case we can write for any $\mathbf{T}$ and $\alpha$

$$
\mathbf{T}(z)=(z-\alpha)^{\operatorname{rord}_{\alpha} T} \cdot \mathbf{R}_{1}(z) \text { and } \mathbf{T}(z)=\mathbf{R}_{2}(z) \cdot(z-\alpha)^{\operatorname{cord}_{\alpha} \mathbf{T}},
$$


where the rows of $\mathbf{R}_{1}$ and the columns of $\mathbf{R}_{2}$ corresponding to an $\alpha$-order $\neq+\infty$ are unique power series around $\alpha$ with a non-zero value at $\alpha$. The decomposition of (1) is then made unique by putting the remaining rows of $\mathbf{R}_{1}$ and the remaining columns of $\mathbf{R}_{2}$ equal to zero. We refer to (1) as the $\alpha$-row and $\alpha$-column representation of $\mathbf{T}$.

Definition 2 The projection operator $\Pi_{\alpha, \sigma}$ acting on $\mathbf{T} \in \mathbf{F}^{p \times q}((z))_{\alpha}$ is defined by

$$
\Pi_{\alpha, \sigma} \mathbf{T}:=\sum_{k=\tau}^{\sigma-1} T_{k}(z-\alpha)^{k}, \quad \text { where } \mathbf{T}(z)=\sum_{k=\tau}^{\infty} T_{k}(z-\alpha)^{k} .
$$

$\Pi_{\alpha, \sigma} \mathbf{T}$ will be called the $(\alpha, \sigma)$ main part of $\mathbf{T}$.

The rational interpolation problem to be considered here is the following: Given $\mathbf{T}_{\alpha} \in \mathbb{F}^{p \times q}((z))_{\alpha}, \forall \alpha \in I$, find a rational function $Z \in \mathbb{F}^{p \times q}(z)$ such that the $(\alpha, \sigma(\alpha))$ main parts of $Z$ and $\mathbf{T}_{\alpha}$ are the same $\forall \alpha \in I$. However, this problem has an infinite number of solutions and as such we only look for those rational functions which are in some sense solutions of minimal complexity. Complexity of a matrix rational function can be defined in several ways. In our case we use the notion of a $\vec{s}$-McMillan degree [12].

DEFINITION 3 Let $\vec{s}=\left(s_{1}, s_{2}, \ldots, s_{p+q}\right)$ with each (shift parameter) $s_{i} \in \mathbf{Z}$. The $\vec{s}$-McMillan degree of a pair of matrix polynomials $(N, D)$, with $N \in \mathbb{F}^{p \times q}[z]$ and $D \in \mathbb{F}^{q \times q}[z]$, is defined as

$\vec{s}-\mathrm{MM}-\operatorname{deg}(N, D)=\max \left\{\operatorname{deg} \operatorname{det} P: P\right.$ is a $q \times q$ submatrix of $\left.z^{\vec{b}} \cdot\left[\begin{array}{l}N \\ D\end{array}\right]\right\}$.

For any rational function $Z \in \mathbb{F}^{p \times q}(z)$ let $Z=N \cdot D^{-1}$ be a right coprime polynomial matrix fraction description (RCPMFD). Then the $\vec{s}-M c$ Millan degree of $Z$ is defined as

$$
\vec{s} \text {-MM-deg } Z=\vec{s} \text {-MM-deg }(N, D) \text {. }
$$

Note that the RCPMFD of a rational function is unique up to a right unimodular factor $U$ of both numerator and denominator [7, p. 441, Th. 6.5-4]); hence the definition of a $\vec{s}$-McMillan degree is well defined. When $\vec{s}=\overrightarrow{0}$ we get the classical McMillan degree. The McMillan degree is a well-known concept in linear system theory. It is a measure of the complexity of a linear system. (see e.g. [7, p. 439]).

The rational problem which we discuss is now given as follows.

Definition 4 Given $\mathbf{T}_{\alpha} \in \mathbb{F}^{p \times q}((z))_{\alpha}, \forall \alpha \in I$, a vector $\vec{s} \in \mathbf{Z}^{(p+q) \times 1}$ and the order indices $\sigma(\alpha)$, find a $Z \in \mathbb{F}^{p \times q}(z)$ with minimal $\vec{s}-M c M i l l a n$ degree such that

$$
\operatorname{ord}_{\alpha}\left(\mathbf{T}_{\alpha}-Z\right) \geq \sigma(\alpha) \quad \forall \alpha \in I \text {. }
$$

We will show that the original rational interpolation problem given some formal Laurent series is equivalent to a rational interpolation problem with the same interpolation points $\alpha \in I$ but given formal power series (no poles anymore). 
3. $(\alpha, \sigma)$-characteristics

Since the rational interpolation problem studied in this paper only requires the $(\alpha, \sigma)$ main part of given formal Laurent series, consideration will have to be taken when we define characteristics limited to this main part.

Definition 5 A formal (matrix) Laurent series $\mathbf{T} \in \mathbf{F}^{p \times q}((z))_{\alpha}$ is called $\alpha$-column reduced iff in the $\alpha$-column representation $\mathbf{T}(z)=\mathbf{R}(z) \cdot(z-\alpha)^{\operatorname{cord}_{\alpha} \mathbf{T}}$ the nonzero columns of $\mathbf{R}(\alpha)$ are linearly independent. $\mathbf{T} \in \mathbb{F}^{p \times q}((z))_{\alpha}$ is called $(\alpha, \sigma)$-column reduced iff $\Pi_{\alpha, \sigma} \mathrm{T}(z)$ is $\alpha$-column reduced.

Clearly if $\mathbf{T}$ is $(\alpha, \sigma)$-column reduced then so is its Laurent polynomial counterpart $\Pi_{\alpha, \sigma} \mathbf{T}$.

Definition 6 Given a formal (matrix) Laurent series $\mathbf{T} \in \mathbb{F}^{p \times q}((z))_{\alpha}$, a matrix polynomial $D_{\mathrm{T}} \in \mathbb{F}^{q \times q}[z]$ is called an $(\alpha, \sigma)$-column reducing denominator of $\mathrm{T}$ iff

(a) $\operatorname{det} D_{\mathrm{T}}(\alpha) \neq 0$ and hence $D_{\mathrm{T}}^{-1} \in \mathbb{F}^{q \times q}[[z]]_{\alpha}$,

(b) $\mathbf{T} \cdot D_{\mathbf{T}}$ is $(\alpha, \sigma)$-column reduced.

Note that an $(\alpha, \sigma)$-column reduced denominator does not depend on $\mathbf{T}$ but only on $\Pi_{\alpha, \sigma} \mathbf{T}$. This can be verified using the following lemma.

LEMMA 1 For any matrix Laurent series $\mathbf{T}_{1}$ around $\alpha$ and any matrix power series $\mathbf{T}_{2}$ around $\alpha$, both of suitable size, we have

$$
\Pi_{\alpha, \sigma}\left(\mathbf{T}_{1} \cdot \mathbf{T}_{2}\right)=\Pi_{\alpha, \sigma}\left(\Pi_{\alpha, \sigma}\left(\mathbf{T}_{1}\right) \cdot \mathbf{T}_{2}\right) .
$$

Proof: Trivial.

Moreover, as seen in the following Theorem, there exist further invariances, namely so-called $(\alpha, \sigma)$-characteristics.

TheOREM 1 Let $\mathbf{T} \in \mathbb{F}^{p \times q}((z))_{\alpha}$, with corresponding $(\alpha, \sigma)$-column reduced denominator $D_{\mathrm{T}} \in \mathbb{F}^{q \times q}[z]$ and $\alpha$-column representation

$$
\Pi_{\alpha, \sigma}\left(\mathbf{T} \cdot D_{\mathbf{T}}\right)=N_{\mathbf{T}} \cdot(z-\alpha)^{\vec{\delta}(\mathbf{T})} .
$$

Then the entries of $\vec{\delta}(\mathrm{T})$ are unique up to permutation.

Proof: Suppose $D_{1}, D_{2}$ are two $(\alpha, \sigma)$-reducing denominators of $\mathrm{T}$, having the $\alpha$-column representations

$$
\Pi_{\alpha, \sigma}\left(\mathbf{T} \cdot D_{1}\right)=N_{1} \cdot(z-\alpha)^{\vec{\sigma}_{1}}, \quad \text { and } \quad \Pi_{\alpha, \sigma}\left(\mathbf{T} \cdot D_{2}\right)=N_{2} \cdot(z-\alpha)^{\vec{\sigma}_{2}} .
$$

Without loss of generality we may assume that the columns of $D_{1}, D_{2}$ are ordered in such a manner that the components of both $\vec{\delta}_{1}$ and $\vec{\delta}_{2}$ are ascending. Suppose 
that there exists a $k$ with $\delta_{1, j}=\delta_{2, j}, j=q, q-1, \ldots, k$ but $\delta_{1, k-1}>\delta_{2, k-1}$. Since $D_{1}(\alpha)$ is regular, we can form $R:=D_{1}^{-1} \cdot D_{2}$, a (matrix) power series about $\alpha$. Set $\Lambda_{j}(z):=(z-\alpha)^{\vec{\delta}_{j}}, j=1,2$. Then using Lemma 1 we have

$$
\begin{aligned}
N_{2} \cdot \Lambda_{2} & =\Pi_{\alpha, \sigma}\left(\mathbf{T} \cdot D_{2}\right)=\Pi_{\alpha, \sigma}\left(\mathbf{T} \cdot D_{1} \cdot D_{1}^{-1} \cdot D_{2}\right) \\
& =\Pi_{\alpha, \sigma}\left(\Pi_{\alpha, \sigma}\left(\mathbf{T} \cdot D_{1}\right) \cdot D_{1}^{-1} \cdot D_{2}\right)=\Pi_{\alpha, \sigma}\left(N_{1} \cdot \Lambda_{1} \cdot R\right) .
\end{aligned}
$$

Since $N_{j} \cdot \Lambda_{j}=\Pi_{\alpha, \sigma}\left(N_{j} \cdot \Lambda_{j}\right), j=1,2$ are both $(\alpha, \sigma)$-column reduced, Definition 5 implies that $R(\alpha)$ has the following form

$$
R(\alpha)=\left[\begin{array}{ccc}
R_{11} & 0 & 0 \\
R_{21} & 0 & 0 \\
R_{31} & R_{3,2} & R_{3,3}
\end{array}\right]
$$

where the rows and columns are partitioned as: $k-2,1, q-k+1$. But $R$ is regular at $\alpha$ because $D_{1}(\alpha)$ and $D_{2}(\alpha)$ are both regular, a contradiction.

DEFINITION 7 The entries of $\vec{\delta}(\mathbf{T})$ defined in the previous theorem are called the $(\alpha, \sigma)$-characteristics ${ }^{1}$ of $\mathbf{T}$. The number of finite $(\alpha, \sigma)$-characteristics is called the $(\alpha, \sigma)$-index ${ }^{2}$ and denoted as $\kappa_{\alpha, \sigma}(\mathrm{T})$. In addition, the sum of the negative $(\alpha, \sigma)$ characteristics of $\mathbf{T}$ is called the $(\alpha, \sigma)$-pole multiplicity and will be abbreviated as $\Delta(\alpha, \sigma, \mathbf{T})$.

The dependency of $(\alpha, \sigma)$-characteristics of $\mathbf{T}$ on $\sigma$ can be described explicitly.

Theorem 2 Let $\mathbf{T} \in \mathbb{F}^{p \times q}((z))_{\alpha}, \sigma_{1}<\sigma_{2}$ and $\vec{\delta}_{1}$ the $\left(\alpha, \sigma_{1}\right)$-characteristics and $\vec{\delta}_{2}$ the $\left(\alpha, \sigma_{2}\right)$-characteristics of $\mathbf{T}$, respectively. Then the entries of $\vec{\delta}_{1}$ and $\vec{\delta}_{2}$ smaller than $\sigma_{1}$ are unique up to permutation.

Proof: Let $D_{\mathrm{T}}$ be an $\left(\alpha, \sigma_{2}\right)$-reducing denominator of $\mathbf{T}$, then (after suitable permutation of columns of $D_{\mathrm{T}}$ ) we may write

$$
\Pi_{\alpha, \sigma_{2}}\left(\mathbf{T} \cdot D_{\mathbf{T}}\right)=\left[\begin{array}{ll}
N_{\mathbf{T}}^{-} & N_{\mathrm{T}}^{+}
\end{array}\right] \cdot\left[\begin{array}{cc}
(z-\alpha)^{\vec{\delta}^{-}} & 0 \\
0 & (z-\alpha)^{\vec{b}^{+}}
\end{array}\right]
$$

where all the entries of $\vec{\delta}_{2}^{-}$are smaller than $\sigma_{1}$ and $\vec{\delta}_{2}^{+}$contains the remaining entries. Applying the projection operator $\Pi_{\alpha, \sigma_{1}}$ gives

$$
\Pi_{\alpha, \sigma_{1}}\left(\mathbf{T} \cdot D_{\mathbf{T}}\right)=\left[\Pi_{\alpha, \sigma_{1}}\left(N_{\mathbf{T}}^{-} \cdot(z-\alpha)^{\vec{\delta}^{-}}\right)(z-\alpha)^{-\vec{\delta}^{-}} \quad 0\right] \cdot\left[\begin{array}{cc}
(z-\alpha)^{\vec{\delta}^{-}} & 0 \\
0 & 0
\end{array}\right]
$$

which is obviously $\alpha$-column reduced. ${ }^{3}$

\footnotetext{
${ }^{1}$ Note the connection with the structural indices from linear system theory: see Kailath [7, p. 447]

${ }^{2}$ This number is bounded by $\min \{p, q\}$, the maximal number of columns of $N_{\mathrm{T}}(\alpha)$ which can be linearly independent.

${ }^{3}$ In particular, we may conclude that an $\left(\alpha, \sigma_{2}\right)$-reducing denominator also is an $\left(\alpha, \sigma_{1}\right)$-reducing denominator for $\sigma_{1}<\sigma_{2}$.
} 
As a consequence of Theorem 2, we may specify some cases where the $(\alpha, \sigma)$-pole multiplicity does not depend on $\sigma$.

Corollary 1 Let $\mathbf{T} \in \mathbb{F}^{p \times q}((z))_{\alpha}$ with $\kappa_{\alpha, \sigma}=\min \{p, q\}$ or $\sigma \geq 0$. Then for any $\mathbf{T}^{\prime}$ with $\operatorname{ord}_{\alpha}\left(\mathbf{T}-\mathbf{T}^{\prime}\right) \geq \sigma$, the $(\alpha, 0)$-characteristics of $\mathbf{T}$ and $\mathbf{T}^{\prime}$ coincide. In particular, the pole multiplicities $\Delta\left(\alpha, \sigma, \mathbf{T}^{\prime}\right), \Delta(\alpha, \sigma, \mathbf{T}), \Delta\left(\alpha, 0, \mathbf{T}^{\prime}\right)$ and $\Delta(\alpha, 0, \mathbf{T})$ all are equal.

Multiplication with $(z-\alpha)^{\tau}$ changes the $\alpha$-characteristics in the following way:

Corollary 2 Let $\mathbf{T}$ have the $(\alpha, \sigma)$-characteristics $\vec{\delta}$ and let $D_{\mathbf{T}}$ be an $(\alpha, \sigma)$ reducing denominator of $\mathbf{T}$. Then for any integer $\tau$, the function $\mathbf{T}_{\tau}:=(z-\alpha)^{\tau} \cdot \mathbf{T}$ has the $(\alpha, \sigma+\tau)$-characteristics $\vec{\delta}+\tau \cdot \vec{e}$ and $D_{\mathrm{T}}$ is an $(\alpha, \sigma+\tau)$-reducing denominator of $\mathbf{T}_{\tau}$.

\section{Construction of $(\alpha, \sigma)$-characteristics}

How can we construct an $(\alpha, \sigma)$-reduced denominator and, hence, the $(\alpha, \sigma)$-characteristics of a given formal Laurent series $\mathbf{T}$ ? The following constructive method generates a finite sequence $\left(N_{k}, D_{k}, V_{k}\right)_{k}$ with

$$
\begin{aligned}
\text { Initialization: } & D_{0}:=I_{q \times q} \\
\text { Recurrence: } & N_{k} \in \mathbb{F}^{p \times q}((z))_{\alpha}, \quad N_{k}:=\Pi_{\alpha, \vec{\sigma}}\left(\mathbf{T} \cdot D_{k}\right) \\
\text { Recurrence: } & D_{k+1}:=D_{k} \cdot V_{k} \in \mathbb{F}^{q \times q}[z], \quad D_{k+1}(\alpha) \text { being regular, }
\end{aligned}
$$

and $N_{k}$ being a Laurent polynomial around $\alpha$. In the second recurence the polynomial factor $V_{k}$ is determined as follows:

If $N_{k}$ is not $\alpha$-column reduced, we know from Definition 5 that $\exists a \in F^{q \times 1}$ with

$$
\mathbf{R}_{k}(\alpha) \cdot a=0 \quad \text { and } \quad N_{k}(z)=\mathbf{R}_{k}(z) \cdot(z-\alpha)^{\operatorname{cord}_{\alpha} N_{h}}
$$

where $a$ has zero entries when the corresponding columns of $\mathbf{R}(\alpha)$ are zero. Let $\operatorname{cord}_{\max } N_{k}$ be equal to the maximum of the (finite) entries of $\operatorname{cord}_{\alpha} N_{k}$ corresponding to nonzero entries of $N_{k}$ and let $j$ be the index of this maximum in $\operatorname{cord}_{\alpha} N_{k}$. Construct the polynomial vector $v \in \mathbb{F}^{q \times 1}[z]$ as

$$
v:=(z-\alpha)^{-\operatorname{cord}_{\alpha} N_{h}+\operatorname{cord}_{\max } N_{h} \cdot \vec{e} a}
$$

with the entries of $v$ corresponding to zero entries of $a$ also equal to zero. In this case we take $V_{k}=\left[e_{1}, e_{2}, \cdots, e_{j-1}, v, e_{j+1}, \cdots, e_{q}\right]$. Note that $V_{k}(\alpha)$ is regular.

If $N_{k}$ is $\alpha$-column reduced, $D_{k}$ has the required properties of an $(\alpha, \sigma)$-column reduced denominator of $\mathbf{T}$. That this process ends after a finite number of steps is clear since at each step we increase the $\alpha$-order of one of the non-zero columns of $N_{k}$ while at the same time the $\alpha$-orders of the nonzero columns of $N_{k}=\Pi_{\alpha, \sigma}\left(N_{k}\right)$ are bounded by $\sigma-1$.

We mention three important aspects of the above construction. First notice that if $\mathrm{T}$ is a power series around $\alpha$, then all $N_{k}$ are matrix polynomials. Secondly, we see that each $V_{k}$ and therefore also each $D_{k}$ may be assumed to be unimodular. The final point is stated in the next theorem. Its proof makes repeated use of Lemma 1. 
THEOREM 3 Let the matrix rational function $Z$ have the $R C P M F D Z=N \cdot D^{-1}$ with matrix polynomials $N \in \mathbb{F}^{p \times q}[z], D \in \mathbb{F}^{q \times q}[z]$, then there holds:

(a) If $Z(\alpha)$ is finite, then the $(\alpha, \sigma)$-characteristics of $Z$ and the $(\alpha, \sigma)$-characteristics of $N$ coincide for all $\sigma$. In addition none of them are negative.

(b) With $\gamma>\operatorname{ord}_{\alpha} \operatorname{det} D$ we have $\kappa_{\alpha, \gamma}(D)=q$, and with $D$ having the $(\alpha, \gamma)$ characteristics $\delta_{1}, \ldots, \delta_{q^{\prime}}, 0, \ldots, 0, q^{\prime}$ minimal, $Z$ has the $(\alpha, 0)$-characteristics $-\delta_{1}$, $\ldots,-\delta_{q^{\prime}},+\infty, \ldots,+\infty$.

Proof: Note that in case (a) it is also true that $D(\alpha)$ is regular. Hence we may initialize the above procedure with $D_{0}=D$, i.e., there exists a unimodular matrix $V \in \mathbb{F}^{q \times q}[z]$ such that $D \cdot V$ is an $(\alpha, \sigma)$-reducing denominator and therefore $\Pi_{\alpha, \sigma}(N \cdot V)$ is $(\alpha, \sigma)$-reduced, with its $\alpha$-column representation yielding the $(\alpha, \sigma)$ characteristics of $Z$.

In order to prove (b), we first notice that $\operatorname{det} D \neq 0$ and so the quantity $\gamma$ is welldefined. Suppose that the unimodular matrix $V$ is an $(\alpha, \gamma)$-reducing denominator of $D$, i.e., there exist two polynomial matrices $D_{0}, D_{0}^{\prime}$ with

$$
D \cdot V=D_{0} \cdot(z-\alpha)^{\vec{\delta}}+(z-\alpha)^{\gamma} \cdot D_{0}^{\prime},
$$

$\vec{\delta}$ containing only nonnegative integers or $+\infty$, and the nonzero columns of $D_{0}$ are linearly independent at $\alpha$. Taking determinants on both sides we see that none of the columns of $D_{0}$ may be different from zero, since otherwise the $\alpha$-order of the right hand side would be greater or equal to $\gamma$ which is greater than $\operatorname{ord}_{\alpha} \operatorname{det}(D \cdot V)=$ $\operatorname{ord}_{\alpha} \operatorname{det} D<\gamma$. Consequently, $\kappa_{\alpha, \gamma}(D)=q$, and we may combine the matrices $D_{0}, D_{0}^{\prime}$ such that without loss of generality $D_{0}^{\prime}=0$. By assumption, the pair $(N, D)$ and therefore the pair $(N \cdot V, D \cdot V)$ is right coprime. The first $q^{\prime}$ columns of $D \cdot V=D_{0} \cdot(z-\alpha)^{\vec{\delta}}$ are zero at $\alpha$, and hence the first $q^{\prime}$ columns of $N \cdot V$ denoted by $N^{+}$must be linearly independent at $\alpha$. We may conclude that $D_{0}$ is an $(\alpha, 0)$-reducing denominator of $Z$ since with $\vec{\delta}^{\prime}:=\left(\delta_{1}, \ldots, \delta_{q^{\prime}}\right)$

$$
\Pi_{\alpha, 0}\left(Z \cdot D_{0}\right)=\Pi_{\alpha, 0}\left(N \cdot V \cdot(z-\alpha)^{-\vec{\delta}}\right)=\left[\Pi_{\alpha, 0}\left(N^{+} \cdot(z-\alpha)^{-\vec{\delta}^{\prime}}\right) 0\right]
$$

being obviously $\alpha$-reduced.

EXAmPle 1 Given $\alpha=0, \sigma=2$, we use the method described above to determine the $(\alpha, \sigma)$-characteristics of

$$
\mathbf{T}=N_{0}=\left[\begin{array}{rr}
1 & -1 \\
0 & 0
\end{array}\right] z^{-1}+\left[\begin{array}{ll}
1 & 2 \\
0 & 0
\end{array}\right] z^{0}+\left[\begin{array}{ll}
0 & 0 \\
0 & 1
\end{array}\right] z^{1}+\cdots .
$$

Because $N_{0}$ is not $\alpha$-column reduced, we multiply to the right by

$$
V_{0}=\left[\begin{array}{ll}
1 & 1 \\
0 & 1
\end{array}\right] \text {. }
$$

We get after projection

$$
N_{1}=\left[\begin{array}{ll}
1 & 0 \\
0 & 0
\end{array}\right] z^{-1}+\left[\begin{array}{ll}
1 & 3 \\
0 & 0
\end{array}\right] z^{0}+\left[\begin{array}{ll}
0 & 0 \\
0 & 1
\end{array}\right] z^{1} .
$$


Because $N_{1}$ still is not $\alpha$-column reduced, we multiply to the right by

$$
V_{1}=\left[\begin{array}{rr}
1 & -3 z \\
0 & 1
\end{array}\right]
$$

We get after projection

$$
N_{2}=\left[\begin{array}{ll}
1 & 0 \\
0 & 0
\end{array}\right] z^{-1}+\left[\begin{array}{ll}
1 & 0 \\
0 & 0
\end{array}\right] z^{0}+\left[\begin{array}{rr}
0 & -3 \\
0 & 1
\end{array}\right] z^{1} .
$$

This last result $N_{2}=\Pi_{\alpha, \sigma}\left(\mathrm{T} \cdot D_{2}\right)$ is $\alpha$-column reduced. Therefore, the $(\alpha, \sigma)$ characteristics are in this case: -1 and 1 , the $(\alpha, \sigma)$-pole multiplicity $\Delta(\alpha, \sigma, \mathbf{T})=$ -1 and the $(\alpha, \sigma)$-index $\kappa_{\alpha, \sigma}=2$.

\section{Equivalence}

Before we can show equivalence between the original rational interpolation problem with pole information and another rational interpolation problem without poles as interpolation points, we need the following theorem.

THEOREM 4 For each matrix rational function $Z \in \mathbb{F}^{p \times q}(z)$ with $(\alpha, 0)$-characteristics $\vec{\delta}$ and for each $\tau \geq 0$ with $\vec{\delta}+\tau \cdot \vec{e} \geq 0$,

$$
\vec{s} \text {-MM-deg } Z=\vec{r} \text {-MM-deg }\left((z-\alpha)^{\tau} \cdot Z\right)-\Delta(\alpha, 0, Z)
$$

with $\vec{r}:=\vec{s}-\tau \cdot(1, \ldots, 1,0, \ldots, 0)$ (q zeros).

Proof: Because $\vec{\delta}_{\tau}:=\vec{\delta}+\tau \cdot \vec{e} \geq 0$, by Corollary 2 the matrix rational function $Z_{\tau}:=(z-\alpha)^{\tau} \cdot Z$ has the $(\alpha, \tau)$-characteristics $\vec{\delta}_{\tau}$, and $Z_{\tau}(\alpha)$ is finite. By the proof of Theorem 3(a) we get a RCPMFD $N_{\tau} \cdot D_{\tau}^{-1}$ for $Z_{\tau}$ with $D_{\tau}$ an $(\alpha, \tau)$-reducing denominator of $Z_{\tau}$, i.e.,

$$
Z_{\tau} \cdot D_{\tau}=N_{\tau}=N_{\tau}^{\prime} \cdot(z-\alpha)^{\vec{\delta}_{\tau}}+(z-\alpha)^{\tau} \cdot N_{\tau}^{\prime \prime}
$$

with $N_{\tau}^{\prime \prime}$ being polynomial. Without loss of generality we may suppose that the components of $\vec{\delta}_{\tau}$ are either smaller than $\tau$ or equal to $+\infty$, and in addition ordered such that with $\vec{\delta}_{\tau}^{-}$containing the finite entries of $\vec{\delta}_{\tau}$ (which then are non-negative and smaller than $\tau$ ) we obtain the consistent partitions

$$
N_{\tau}^{\prime}=\left[\begin{array}{ll}
N_{\tau}^{-} \cdot(z-\alpha)^{\vec{\delta}^{-}} & 0
\end{array}\right], \quad D_{\tau}=\left[\begin{array}{ll}
D_{\tau}^{-} & D_{\tau}^{+}
\end{array}\right]
$$

Notice that the matrix $N_{\tau}^{-}(\alpha)$ by definition of $(\alpha, \tau)$-characteristics has maximal rank, and $D_{\tau}(\alpha)$ is regular, hence also the matrix $D_{\tau}^{+}(\alpha)$ has maximal rank.

A RCPMFD $N \cdot D^{-1}$ for $Z$ can be based upon $N_{\tau}$ and $D_{\tau}$ as follows. Define

$$
\Lambda^{+}:=\left[\begin{array}{cc}
(z-\alpha)^{-\vec{\delta}_{\tau}^{-}} & 0 \\
0 & (z-\alpha)^{-\tau} \cdot I
\end{array}\right], \quad \Lambda^{-}:=\left[\begin{array}{cc}
(z-\alpha)^{\tau \vec{e}-\vec{\delta}_{\tau}^{-}} & 0 \\
0 & I
\end{array}\right]
$$


where $\Lambda^{-}:=(z-\alpha)^{\tau} \cdot \Lambda^{+}$is polynomial. Because $Z=(z-\alpha)^{-\tau} \cdot Z_{\tau}$, a polynomial matrix fraction description of $Z$ is given by the numerator $N:=N_{\tau} \cdot \Lambda^{+}$ and the denominator $D:=D_{\tau} \cdot \Lambda^{-}$. In order to show that $(N, D)$ also is right coprime and therefore a RCPMFD for $Z$, we prove the equivalent condition that $\left[\begin{array}{ll}N(z)^{T} & D(z)^{T}\end{array}\right]^{T}$ has maximal rank $\forall z \in \mathbf{F}$ (cf., [7, Lemma 6.3-6, p. 379]). In case $z \neq \alpha$, this condition follows immediately from the right coprimeness of $\left(N_{\tau}, D_{\tau}\right)$. For $z=\alpha$ we obtain

$$
\left[\begin{array}{l}
N \\
D
\end{array}\right](\alpha)=\left[\begin{array}{l}
N_{\tau} \cdot \Lambda^{+} \\
D_{\tau} \cdot \Lambda^{-}
\end{array}\right](\alpha)=\left[\begin{array}{cc}
N_{\tau}^{-}(\alpha) & 0 \\
X & D_{\tau}^{+}(\alpha)
\end{array}\right]
$$

having also maximal rank (independently of the suitable matrix $X$ ) as shown above.

Hence, for the McMillan degree there holds

$$
\begin{aligned}
\vec{s}-\text { MM-deg } Z & =\vec{s} \text {-MM-deg }\left(N_{\tau} \cdot \Lambda^{+}, D_{\tau} \cdot \Lambda^{-}\right) \\
& =\vec{r} \text {-MM-deg }\left(N_{\tau} \cdot \Lambda^{+} \cdot(z-\alpha)^{\tau}, D_{\tau} \cdot \Lambda^{-}\right) \\
& =\vec{r} \text {-MM-deg }\left(N_{\tau}, D_{\tau}\right)+\operatorname{deg} \operatorname{det} \Lambda^{-} \\
& =\vec{r}-\text { MM-deg } Z_{\tau}+\operatorname{deg} \operatorname{det} \Lambda^{-} .
\end{aligned}
$$

Note that, by Corollary $2, D_{\tau}$ also is an $(\alpha, 0)$-reducing denominator of $Z$, such that $\Delta(\alpha, 0, Z)=-\operatorname{deg} \operatorname{det} \Lambda^{-}$.

Now we are able to show the main assertion.

THEOREM 5 Given the set I of interpolation points $\alpha$, the formal Laurent series $\mathbf{T}_{\alpha}$, the order indices $\sigma(\alpha), \forall \alpha \in I$ and the shift parameters $\vec{s}$. Let $\mathbf{R}_{\alpha}, \rho(\alpha), \tau(\alpha)$, $\forall \alpha \in I, \tau, \omega$ be defined as follows:
(a) choose $\tau_{\alpha}$ such that $-\tau(\alpha) \leq \min \left\{0, \operatorname{ord}_{\alpha}\left(\mathbf{T}_{\alpha}\right)\right\}$,
(b) $\tau:=\sum_{\alpha \in I} \tau(\alpha)$,
(c) $\vec{r}:=\vec{s}-\tau \cdot(1, \ldots, 1,0, \ldots, 0) \quad$ (q zeros),
(d) $\rho(\alpha):=\sigma(\alpha)+\tau(\alpha)$
(e) $\mathbf{R}_{\alpha}:=\omega \cdot \mathbf{T}_{\alpha} \in \mathbb{F}^{p \times q}[[z]]_{\alpha}$ with $\omega:=\prod_{\alpha \in I}(z-\alpha)^{\tau(\alpha)}$,
(f) $\Delta:=\sum_{\alpha \in I} \Delta\left(\alpha, \sigma(\alpha), \mathbf{T}_{\alpha}\right)$.

Moreover, let

$\forall \alpha \in I:$ (a) $\kappa_{\alpha, \sigma(\alpha)}\left(\mathbf{T}_{\alpha}\right)=\min \{p, q\}$

$$
\text { (b) } \sigma(\alpha) \geq 0 \text {. }
$$

(a) For each $Z$ satisfying (2) we have

$$
\vec{s} \text {-MM-deg } Z=\vec{r} \text {-MM-deg }(\omega \cdot Z)-\Delta \text {. }
$$

(b) If $Z_{\tau}$ is a solution to the rational interpolation problem with respect to $\left(\mathbf{R}_{\alpha}, \rho(\alpha)\right.$, $\forall \alpha \in I, \vec{r})$ then $Z:=\frac{1}{\omega} \cdot Z_{\tau}$ is a solution to the rational interpolation problem with respect to $\left(\mathrm{T}_{\alpha}, \sigma(\alpha), \forall \alpha \in I, \vec{s}\right)$.

Proof: By Corollary 1 the $(\alpha, 0)$-characteristics of $\mathbf{T}_{\alpha}$ and $Z$ coincide and $\Delta(\alpha$, $\left.\sigma(\alpha), \mathbf{T}_{\alpha}\right)=\Delta(\alpha, 0, Z)$. Hence, part (a) follows by successively applying Theorem 4. Assertion (b) is an immediate consequence. 
EXAMPLE 2 Take interpolation in 0,1 and -1 with

$$
\sigma(0)=2, \quad \sigma(1)=-1, \quad \sigma(-1)=0, \quad \mathbf{T}_{\alpha}=\mathbf{T}=\left[\begin{array}{cc}
\frac{-1}{(x+1) \cdot(x-1)^{2}} & z-2 \\
z & \frac{1}{(x-1)^{3}}
\end{array}\right]
$$

We can apply Theorem 5 because $\sigma(0) \geq 0$, the negative $(\alpha, \sigma(\alpha))=(1,-1)$ characteristics of $\mathrm{T}$ are -3 and -2 , i.e. they are all known, and $\sigma(-1) \geq 0$. Let us choose the shift parameters $\vec{s}=(1,1,0,0)$. We can take $\tau(0)=0, \tau(1)=3$ and $\tau(-1)=1$. Hence, $\rho(0)=2, \rho(1)=2, \rho(-1)=1, \omega(z)=(z-1)^{3}(z+1)$, $\vec{r}=(-3,-3,0,0)$ and $\mathbf{R}_{\alpha}=\mathbf{R}=\mathbf{T} \cdot \omega, \forall \alpha \in I=\{0,1,-1\}$. To solve the pole-free problem, we use the module-theoretic framework of [12]. A basis for the submodule corresponding to $(\mathbf{R}, \rho(\alpha), \forall \alpha \in I, \vec{r})$ is given by the columns of the polynomial matrix P:

$$
\left[\begin{array}{cccc}
-(3 z-2)(z+1)(z-1)^{2} & -(z-1) & -(z+1)(z-1) & (z+1)(z-1)^{2}(z-2) \\
z+1 & -(z+1)(z-1)^{2} z & -(z+1)(z-1)^{2} z & -(z+1)^{2} \\
0 & -1 & -(z+1) & 0 \\
-1 & 0 & 0 & z+1
\end{array}\right]
$$

with corresponding $\vec{r}$-degrees $(1,1,1,1)$. All solutions $Z_{\tau}$ of the "no-pole" problem having minimal $\vec{r}-M M$-degree 2 can be written as $Z_{\tau}=N_{\tau} \cdot D_{\tau}^{-1}$ with

$$
\left[\begin{array}{l}
N_{\tau} \\
D_{\tau}
\end{array}\right]=\mathbf{P} \cdot\left[\begin{array}{ll}
1 & 0 \\
0 & 1 \\
a & c \\
b & d
\end{array}\right] \quad \text { with } a, b, c, d \in \mathbf{F}
$$

and $D_{\tau}(0)$ regular, i.e. $b \neq 1$ and $c \neq-1, D_{\tau}(1)$ regular, i.e. $b \neq 2$ and $c \neq-2$, $D_{\tau}(-1)$ regular, giving no further conditions on $a, b, c$ or $d$.

Hence, all solutions $Z$ of the "pole" problem can be written as $Z=Z_{\tau} / \omega$ having minimal $\vec{s}-M M$-degree

$$
\vec{s}-\mathrm{MM}-\operatorname{deg} Z=\vec{r}-\mathrm{MM}-\operatorname{deg} Z_{\tau}-\Delta=2-(-3-2-1)=8 .
$$

Note that the equivalence is also valid when we consider vector order indices $\vec{\sigma}(\alpha)$ instead of scalar ones.

CoRollary 3 Consider the rational interpolation problem of Definition \& but with

$$
\operatorname{cord}_{\alpha}\left(\mathbf{T}_{\alpha}-Z\right) \geq \vec{\sigma}(\alpha) \quad \forall \alpha \in I
$$

instead of (2). Take the notation of Theorem 5 except for $\rho(\alpha)$ for which a vector variant is defined as $\vec{\rho}(\alpha):=\vec{\sigma}(\alpha)+\tau \vec{e}$. Instead of (12), we assume that

$\forall \alpha \in I:$

(a) $\kappa_{\alpha, \sigma_{\min }(\alpha)}\left(\mathbf{T}_{\alpha}\right)=\min \{p, q\}$

(b) $\vec{\sigma}(\alpha) \geq 0$ 
with $\sigma_{\min }(\alpha)=\min _{i=1}^{p}\left\{\sigma_{i}(\alpha)\right\} . \Delta$ is defined as

$$
\Delta=\sum_{\alpha \in I} \Delta\left(\alpha, \sigma_{\min }(\alpha), \mathbf{T}_{\alpha}\right) .
$$

In this case, the results of Theorem 5 are also valid.

Proof: The condition (15) guarantees that the $(\alpha, 0)$-indices of $Z$ are fixed.

\section{Open problem}

If condition (12) in Theorem 5 (or (15) of Corollary 3) is not satisfied, then it is an open problem how the original rational interpolation problem can be transformed into one without pole information. As an example we have:

Example 3 Take interpolation in 0 and 1 with

$$
\sigma(0)=2, \quad \sigma(1)=-3, \quad \mathbf{T}_{\alpha}=\mathbf{T}=\left[\begin{array}{cc}
\frac{1}{1-z} & 0 \\
0 & \frac{1}{(1-z)^{8}}
\end{array}\right]
$$

Then we have

$$
\omega(z)=(1-z)^{5}, \quad \tau=5, \quad \rho(0)=2, \quad \rho(1)=2 .
$$

Note that $\mathbf{T}$ has the negative $(1,+\infty)$-characteristics -5 and -1 , but $(1, \sigma(1))$ characteristics -5 and $+\infty$. For the submodule induced by the interpolation conditions due to $\rho(\alpha)$ and $\mathbf{R}_{\alpha}=\omega \cdot \mathbf{T}_{\alpha}$ we have as two bases the columns of the matrices

$$
\begin{gathered}
\mathbf{P}_{1}=\left[\begin{array}{cccc}
z^{2}(1-z)^{2} & 0 & (1-z)^{4} & 0 \\
0 & z^{2}(1-z)^{2} & 0 & 1+5 z \\
0 & 0 & 1 & 0 \\
0 & 0 & 0 & 1
\end{array}\right], \\
\mathbf{P}_{2}=\left[\begin{array}{cccc}
z^{2}(1-z)^{2} & 0 & (1-z)^{2}(1-2 z) & 0 \\
0 & z^{2}(1-z)^{2} & 0 & 1+5 z \\
0 & 0 & 1 & 0 \\
0 & 0 & 0 & 1
\end{array}\right] .
\end{gathered}
$$

(a) Take $\vec{s}=(1,1,0,0)$ and therefore $\vec{r}=(-4,-4,0,0)$. Then both bases $\mathbf{P}_{1}$ and $\mathrm{P}_{2}$ are $\vec{r}$-column-reduced. We obtain the two "no-pole" solutions with minimal $\vec{r}$-McMillan degree

$$
Z_{1}^{\prime}=\mathbf{R}=\left[\begin{array}{cc}
(1-z)^{4} & 0 \\
0 & 1+5 z
\end{array}\right], \quad Z_{2}^{\prime}=\left[\begin{array}{cc}
(1-z)^{2}(1-2 z) & 0 \\
0 & 1+5 z
\end{array}\right]
$$

which yields for our "pole-problem" the two solutions

$$
\begin{array}{r}
Z_{1}=\frac{1}{\omega} \cdot Z_{1}^{\prime}=\mathrm{T} \text { with } \vec{s}-\mathrm{MM}-\operatorname{deg} Z_{1}=\vec{r}-\mathrm{MM}-\operatorname{deg} Z_{1}^{\prime}+6=0+6=6 \\
Z_{2}=\frac{1}{\omega} \cdot Z_{2}^{\prime} \text { with } \vec{s}-\mathrm{MM}-\operatorname{deg} Z_{2}=\vec{r}-\mathrm{MM}-\operatorname{deg} Z_{2}^{\prime}+8=0+8=8
\end{array}
$$


where we have applied Theorem 4 for computing the McMillan-degree. We see that two solutions of the "no-pole-problem" with minimal $\vec{r}$-MM-degree lead to solutions of the "pole-problem" with different $\vec{s}$-MM-degrees, $Z_{1}$ having minimal $\vec{s}$-MM-degree.

(b) Take $\vec{s}=(2,2,0,0)$ and therefore $\vec{r}=(-3,-3,0,0)$. Then only the basis $\mathbf{P}_{2}$ is $\vec{r}$-column-reduced. We obtain the two "no-pole" solutions $Z_{1}^{\prime}$ and $Z_{2}^{\prime}$ of (18), but

$$
\begin{gathered}
Z_{1}=\frac{1}{\omega} \cdot Z_{1}^{\prime}=\mathrm{T} \text { with } \vec{s} \text {-MM-deg } Z_{1}=\vec{r}-\mathrm{MM}-\operatorname{deg} Z_{1}^{\prime}+6=1+6=7 \\
Z_{2}=\frac{1}{\omega} \cdot Z_{2}^{\prime} \text { with } \vec{s} \text {-MM-deg } Z_{2}=\vec{r} \text {-MM-deg } Z_{2}^{\prime}+8=0+8=8 .
\end{gathered}
$$

Here the solution $Z_{1}$ of the "pole-problem" with minimal $\vec{s}$-MM-degree does not result from a solution of the "no-pole-problem" with minimal $\vec{r}$-MM-degree.

\section{Conclusion}

In this paper, we have shown that the matrix rational interpolation problem with poles as interpolation points can be transformed into a "pole-free" problem if enough pole-information is known. As a result, all solutions of the original problem having minimal complexity can be written down.

However, when not enough pole-information is available, it remains an open problem how we can represent all matrix rational functions satisfying the interpolation conditions.

\section{References}

1. B. Beckermann. A reliable method for computing M-Padé approximants on arbitrary staircases. J. Comput. Appl. Math., 40:19-42, 1990.

2. B. Beckermann and G. Labahn. A uniform approach for the fast computation of Matrix-type Padé approximants. SIAM J. Matrix Anal. Appl. To appear.

3. B. Beckermann and G. Labahn. A uniform approach for Hermite-Padé and simultaneous Padé approximants and their matrix-type generalizations. Numerical Algorithms, 3:45-54, 1992.

4. S. Cabay, G. Labahn, and B. Beckermann. On the theory and computation of non-perfect Padé-Hermite approximants. J. Comput. Appl. Math., 39:295-313, 1992.

5. M.H. Gutknecht. Continued fractions associated with the Newton-Padé table. Numer. Math., 56:547-589, 1989.

6. M.H. Gutknecht. The rational interpolation problem revisited. Rocky Mountain J. Math., 21(1):263-280, 1991.

7. T. Kailath. Linear Systems. Prentice-Hall, 1980.

8. H. Stahl. Existence and uniqueness of rational interpolants with free and prescribed poles. In E.B. Saff, editor, Approximation Theory, Tampa, 1985-1986, pages 180-208. Springer, 1987.

9. M. Van Barel and A. Bultheel. A new approach to the rational interpolation problem. J. Comput. Appl. Math., 32(1-2):281-289, 1990.

10. M. Van Barel and A. Bultheel. A new approach to the rational interpolation problem: the vector case. J. Comput. Appl. Math., 33(3):331-346, 1990.

11. M. Van Barel and A. Bultheel. The computation of non-perfect Padé-Hermite approximants. Numer. Alg., 1:285-304, 1991.

12. M. Van Barel and A. Bultheel. A general module theoretic framework for vector M-Padé and matrix rational interpolation. Numer. Alg., 3:451-461, 1992.

13. M. Van Barel and A. Bultheel. A new formal approach to the rational interpolation problem. Numer. Math., 62:87-122, 1992. 